\title{
Association between the XRCC1 Arg194Trp Polymorphism and Glioma Risk: an Updated Meta-analysis
}

\author{
Cheng $\mathrm{Xu}^{1,2 \&}$, Pin Chen ${ }^{3 \&}$, Wei Liu ${ }^{1,2}$, Ai-Hua Gu ${ }^{1,2 *}$, Xin-Ru Wang ${ }^{1,2}$
}

\begin{abstract}
Gliomas are the most common type of primary brain tumors. The XRCC1 Arg194Trp variant affects the proliferating cell nuclear antigen( PCNA) binding region, which suggests that this mutation may contribute to gliomagenesis and a number of articles have examine the association between XRCC1 Arg194Trp and the susceptibility to glioma. However, the results were conflicting. Test of heterogeneity, sensitivity analysis, metaanalysis, and assessment of publication bias were all performed in our present meta-analysis, covering a total of 5,407 patients and 7,715 healthy persons. In the overall analysis the XRCC1 Arg194Trp polymorphism showed a significant association with glioma susceptibility in a recessive mode l(for TrpTrp $v s$ ArgArg+ArgTrp: $O R=1.918$, 95\% $\mathrm{CI}=1.575-2.336, \mathrm{I}^{2}=2.3 \%$ ). In addition, analysis of subgroups presented an increased risk in Asians and populations-based on hospitals. The results suggested that the XRCC1 Arg194Trp polymorphism is a genetic risk factor for glioma, especially in Asian population. To further evaluate gene-gene and gene-environment interactions on XRCC1 polymorphisms and glioma risk, thousands of subjects and tissue-specific biochemical characterizations are required.
\end{abstract}

Keywords: XRCC1 - Arg 194Trp polymorphism - glioma - meta analysis

Asian Pac J Cancer Prev, 15 (17), 7419-7424

\section{Introduction}

Gliomas are the most common type of primary brain tumors (Ricard et al., 2012), with an incidence rate of approximately 6/100,000 per year worldwide. Despite the advances in neurosurgery and chemotherapy, median survival of only 12 to 15 months among patients in the United States with glioblastoma, the most common type of glioma (Wen et al., 2008). Nowadays, the cause of glioma is still unknown and the etiology has been poorly understood, and may be multifactorial resulting from the interaction of intrinsic and environmental factors (Connelly et al., 2007, Bondy et al., 2008). The only established environmental risk factor is exposure to therapeutic or high-dose ionizing radiation (Schwartzbaum et al., 2006; Bondy et al., 2008; Ostrom et al., 2011).

$\mathrm{X}$-ray repair cross complementing group 1 (XRCC1) acts as a scaffolding protein that functions in the repair of base excision and DNA single-strand breaks, the two most common repair pathways in cellular DNA (Caldecott et al., 1995). XRCC1 interacts with a number of proteins crucial to the BER/SSBR pathways, including OGG1, NEIL2, NTH1, MPG, UNG2, AP endonuclease-1 (APE-1), poly (ADPribose) polymerase, DNA polymerase $\beta$, and DNA ligase 3 (Caldecott et al., 1995; Dianov et al., 1999; Thompson et al., 2000; Vidal et al., 2001; Marsin et al., 2003; Campalans et al., 2005; Akbari et al., 2010). Eight non synonymous coding single nucleotide polymorphisms were existed in $X R C C 1$, three were related to glioma in former extensively studies. These are: Arg194Trp (R194W, rs1799782, exon 6), Arg280His (R280H, rs25489, exon 9) and Arg399Gln (R399Q, rs25487, exon 10). Among them, the XRCC1 Arg194Trp variant located in the proliferating cell nuclear antigen (PCNA) binding region, which suggests that this mutation may be result in gliomagenesis. However, these studies have failed to yield a consistent conclusion (Kiuru et al., 2008; Liu et al., 2009; McKean-Cowdin et al., 2009; Rajaraman et al., 2010; Custodio et al., 2011; Hu et al., 2011; Zhou et al., 2011; Liu et al., 2012; Luo et al., 2013; Pan et al., 2013; Wang et al., 2012; Xu et al., 2013).

Recently, Jiang et al. (2013) reported that XRCC1 Arg194Trp polymorphism might have no influence on the susceptibility of glioma; However, only four literatures were included in this meta-analysis. Subsequently seven molecular epidemiologic studies on the association between this polymorphism and glioma risk also presented contradictory results. Here, we update previous metaanalyses, with additional data to assess the effect of XRCC1 Arg194Trp polymorphism on glioma incidence. In this meta-analysis, we aimed to obtain outline risk evaluates for the XRCC1 Arg194Trp associated with glioma risk.

${ }^{1}$ State Key Laboratory of Reproductive Medicine, Institute of Toxicology, ${ }^{2}$ Key Laboratory of Modern Toxicology of Ministry of Education, School of Public Health, Nanjing Medical University, ${ }^{3}$ Department of Neurosurgery, The First Affiliated Hospital, Nanjing Medical University, Nanjing,China ${ }^{\star} E q u a l$ contributors*For correspondence: aihuagu@njmu.edu.cn 


\section{Materials and Methods}

We searched the electronic databases Web of Science, PubMed and EMBASE using such terms ("glioma" or "gliomas" or "brain cancer"), ("XRCCl" or "X-ray repair cross-complementation group 1" or "DNA repair gene" and "polymorphism or variant or variation") (last search was updated on February12, 2014).

The inclusion criteria of this meta-analysis were: 1) XRCC1 Arg194Trp polymorphism and glioma; 2) sufficient maternal genotype data for estimating an odds ratio (OR) with a $95 \%$ confidence interval $(\mathrm{CI})$; and 3) published in English. The criteria for the exclusion of studies are as follows: 1) not relate to the XRCC1 Arg 194Trp polymorphism and glioma;2) not a primary case-control study; 3 ) no usable or sufficient maternal genotype data reported.

\section{Data collection}

The first author, publication year, country of origin, ethnicity, sources of controls, genotyping method, frequency of Trp-allele in controls, number of genotyped cases and controls were collected independently by two authors (XC and $\mathrm{CP})$ in Table 1.

Odds ratio (OR) plus 95\% CIs was used to calculate the strength of association between glioma risk and the XRCC1 Arg194Trp polymorphism. The pooled ORs were computated for the additive model (Trp versus Arg), homozygote comparison (TrpTrp versus ArgArg), heterozygote comparison (ArgTrp versus ArgArg), dominant model (ArgTrp+TrpTrp versus ArgArg) and recessive model (TrpTrp versus ArgArg+ArgTrp).

\section{Statistical methods}

First, we assessed HWE for the controls in each study. $\mathrm{X}^{2}$ test of heterogeneity was calculated in comparison with pooled articles, when $p$ value was $>0.10$ we used fixed-effects model (Mantel-Haenszel); In contrast, the random-effects models (DerSimonian and Laird) was used. Subgroup analyses were also conducted by ethnicity, study design, genotyping method and HWE. Also, the sensitivity analyses and publication bias was performed. In brief each time a single article was removed, then we analysed remain articles respectively. The methods of Egger et al. and Begg et al. were to test the publication bias. The result consists of the Begg's funnel plot and
Egger's test. All statistical analyses were performed using the STATA software version 11 (Stata Corporation, College Station, TX). Two-sided P values less than 0.05 were considered statistically significant

\section{Results}

\section{Literatures}

The characteristics of the selected studies are listed in Table1.There twenty-one studies were meet our search terms, and 12 eligible studies were finally included in. Totally, 5407 patients and 7715 healthy persons were for meta-analysis. Among our analysis seven studies of Study population were Asians and four were Caucasians, three studies were population-based controls and eight studies were hospital-based controls. The distributions of the genotypes in the control groups in 7 studies were not in HWE. All of included articles were able to analyse for the allel model, additive model, dominant model and recessive model. The major baseline characteristics of the 12 eligible publications were reported in Table 1.

\section{Meta analysis}

Overall, the Trp194 allel was 15.6\% (95\%CI, 9.9-21.3) among all over the glioma, which was between Caucasians and Asian. There were significant differences in terms of the variant Trp194 allele frequency between the only two ethnicities [Caucasians, 5.3\%; 95\% confidence interval (95\%CI), 0-10.6; Asian, 19.3\%; 95\%CI, 15.6-23.0; $p=0.0002$, Figure. 1$]$

\section{Allel Frequencies of XRCC1 Arg194Trp Among Controls}

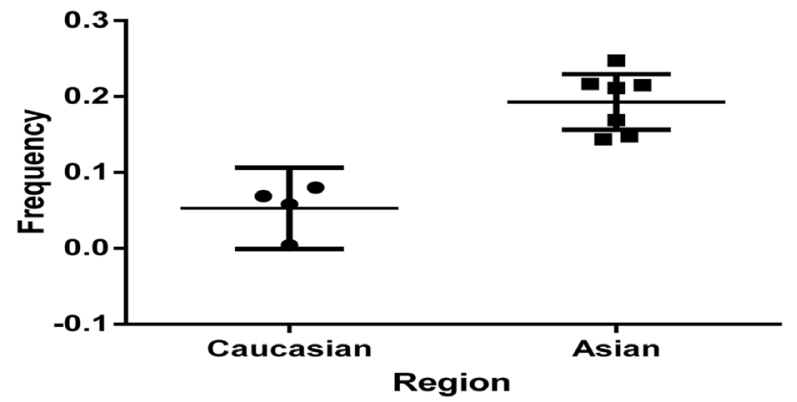

Figure 1. Allele Frequencies and their $95 \%$ CIs of the XRCC1 Arg194Trp Polymorphism among Control Subjects by Different Ethnicity. Each data point represents a separate article for the indicated association

Table 1. Characteristics of the Association Studies on XRCC1 Arg194Trp Polymorphism and the Risk of Glioma

\begin{tabular}{|c|c|c|c|c|c|c|c|c|c|c|}
\hline First author & Year & Country & Ethnicity & Cancertype & $\begin{array}{c}\text { Source } \\
\text { of controls }\end{array}$ & $\begin{array}{c}\text { Genotyping } \\
\text { method }\end{array}$ & Cases & Controls & $\begin{array}{l}\text { Frequency } \\
\text { rp-allele in cor }\end{array}$ & HWE \\
\hline Kiuru & 2008 & Eropean & Caucasia & glioma & popilation & PCR-PFLP & 700 & 1556 & 0.06 & $\mathrm{~N}$ \\
\hline Liu & 2009 & USA & Caucasia & glioma & popilation & massARRA & 210 & 365 & 0.004 & $\mathrm{~N}$ \\
\hline Mckean & 2009 & USA & Caucasia & glioma & mixed & Taqman & 962 & 1922 & 0.07 & $\mathrm{~N}$ \\
\hline Rajaraman & 2010 & USA & Caucasia & glioma & mixed & Taqman & 342 & 468 & 0.08 & $\mathrm{~N}$ \\
\hline Zhou & 2011 & China & Asian & glioma & hospital & PCR-PFLP & 271 & 289 & 0.25 & $\mathrm{~N}$ \\
\hline $\mathrm{Hu}$ & 2011 & China & Asian & glioma & hospital & PCR-CTPP & 127 & 249 & 0.22 & $\mathrm{Y}$ \\
\hline Custódio & 2011 & Brasil & mixed & glioma & popilation & PCR-PFLP & 80 & 100 & 0.31 & $\mathrm{Y}$ \\
\hline Wang & 2012 & China & Asian & glioma & hospital & PCR-PFLP & 624 & 580 & 0.21 & $\mathrm{~N}$ \\
\hline Liu & 2012 & China & Asian & glioma & hospital & MassARRAY & 444 & 442 & 0.14 & Y \\
\hline Luo & 2013 & China & Asian & glioma & hospital & PCR-PFLP & 317 & 415 & 0.17 & Y \\
\hline Pan & 2013 & China & Asian & glioma & hospital & MassARRAY & 444 & 443 & 0.15 & Y \\
\hline $\mathrm{Xu}$ & 2013 & China & Asian & glioma & hospital & PCR-PFLP & 886 & 886 & 0.22 & $\mathrm{~N}$ \\
\hline
\end{tabular}


$\operatorname{Arg} A r g$ or Arg genotype was as reference group in our meta-analysis. All ORs and 95\%CIs were in Table 2. In short, among pooled analysis XRCC1 Arg194Trp polymorphism shown a significant association with glioma susceptibility (for Trp vs Arg: OR=1.259, 95\% CI=1. 045-1.517, $\mathrm{I}^{2}=81.7 \% 1$; for TrpTrp $v$ s ArgArg: OR=2.108, 95\% CI $=1.593-2.789, \mathrm{I}^{2}=38.2 \%$; for ArgTrp vs ArgArg: OR=1.106, 95\%CI=0.901-1.359, $\mathrm{I}^{2}=76.0 \%$; for TrpTrp vs ArgArg+ArgTrp: $\mathrm{OR}=1.918,95 \% \mathrm{CI}=1.575-2.336$, $\mathrm{I}^{2}=2.3 \%$; for ArgTrp+TrpTrp vs Arg Arg: OR=1.230, $\left.95 \% \mathrm{CI}=0.997-1.519, \mathrm{I}^{2}=80.1 \%\right)$. The forest plot of dominant model and recessive model result were shown in Figure 2.

\section{Subgroup analysis}

The similar association was discovered in the subgroup analyses. In the subgroup analyses were based on Ethnicity and sources of control. The results were robust, which

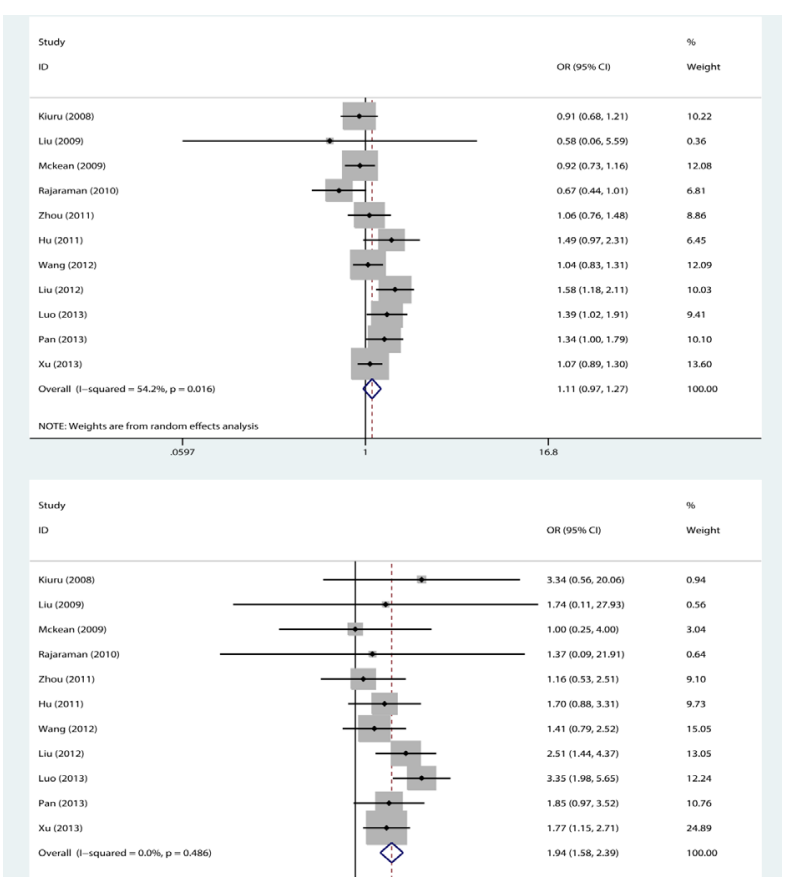

Figure 2. Forest Plot of Glioma Risk Associated with the XRCC1 Arg194Trp Polymorphism (upper: ArgTrp+TrpTrp vs ArgArg; under: TrpTrp vs ArgArg+ArgTrp) in Overall Populations

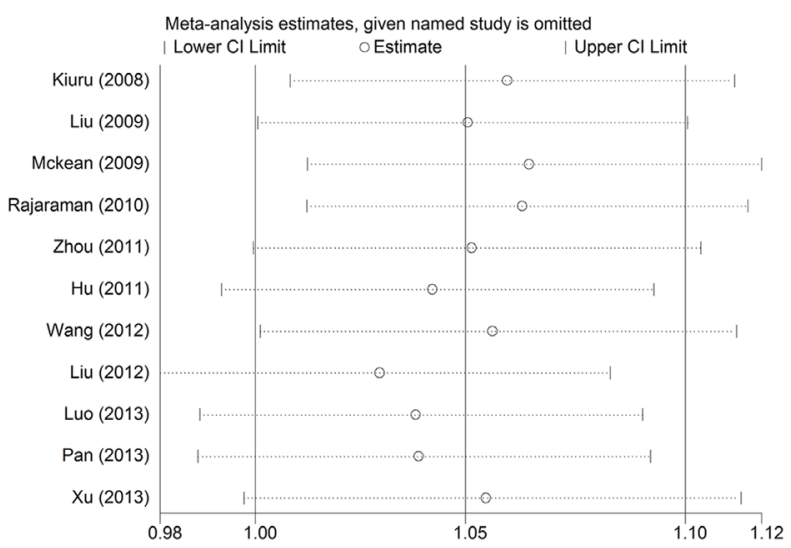

Figure 3. Outlier Analysis for the XRCC1 Arg194Trp Polymorphism Illustrating the Influence of Each Study on Pooled OR did not vary materially after we excluded the study with controls not in HWE. Following significant results were to describe.

Among subgroup of ethnicity, only in Asian existed significant results were in following genetic models : additive model (for Trp $v s$ Arg: $\mathrm{OR}=1.150,95 \% \mathrm{CI}=1.063$ 1.244, $\left.\mathrm{I}^{2}=70.5 \%\right)$, TrpTrp vs ArgArg: $\mathrm{OR}=1.375$, 95\% CI $=1.264-1.495, \mathrm{I}^{2}=41.5 \%$ and recessive model (for TrpTrp $v s$ ArgArg+ArgTrp: OR=1.359, 95\% CI=1.2121.524, $\mathrm{I}^{2}=45 \%$ ) dominant model (for ArgTrp+TrpTrp $v s$ ArgArg: OR=1.098, 95\% CI=1.043-1.156, $\mathrm{I}^{2}=39.6 \%$ ), respectively. While in Caucasian it suggested that XRCC1 Arg 194Trp polymorphism was no association with glioma. As well in populaiton-based on controls, similar significant results were in population-based on controls from hospital. The detailed information was in Table 2. Additionally, when the Genotyping method was MassARRAY, all statistic models presented significantly increased risks.

\section{Test for heterogeneity, sensitivity analyses and publication} bias

Pooled comparisons and subgroup analyses were examined the heterogeneity. In allel and dominant models, among pooled analysis the heterogeneity of $P$ values were all $<0.1$, the results were shown in Table 2 . Therefore, we performed the source of heterogeneity among Ethnicity, sources of control, genotyping method and HWE. When we performed the sensitivity analyses, no matter overall analyses and the subgroup analyses ORs was not altered, suggesting that our results were stability and liability statistically. Also the sensitivity result was in Figure 3. We conducted the Begg's funnel plot and Egger's test to test the publication bias of the eligible studies. The result showed no significant evidence of publication bias (for dominant model $\mathrm{t}=1.22, p=0.249$; for recessive model $\mathrm{t}=-1.03, p=0.328)$. The Begg's funnel plot Figure was in Figure 4.

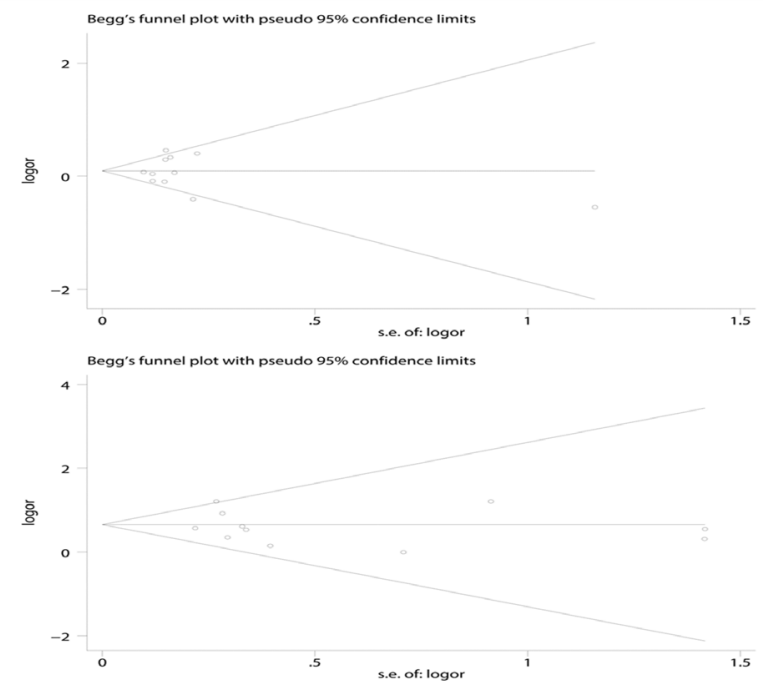

Figure 4. Begg's Funnel Plot of Glioma Risk Associated with the XRCC1 Arg194Trp Polymorphism (upper: ArgTrp+TrpTrp vs ArgArg; Under: TrpTrp vs ArgArg+ArgTrp) in Overall Populations. Each Point Represents a Separate Study for the Indicated Association 
Cheng Xu et al

Table 2. Summary of Comparisons for XRCC1 Arg194Trp Polymorphism and Risk of Glioma

\begin{tabular}{|c|c|c|c|c|c|c|}
\hline model & Variable & Comparisons & OR & $95 \% \mathrm{CI}$ & $P_{h}^{*}$ & $\mathrm{I}^{2}$ \\
\hline \multirow[t]{11}{*}{ Trp vs Arg } & Overall & 12 & 1.259 & $1.045-1.517$ & 0 & $81.70 \%$ \\
\hline & Overall in HWE & 5 & 1.768 & $1.372-2.280$ & 0.005 & $73.20 \%$ \\
\hline & Ethnicity & & & & & \\
\hline & Asian & 7 & 1.15 & $1.063-1.244$ & 0.002 & $70.50 \%$ \\
\hline & Caucasian & 4 & 0.921 & $0.826-1.026$ & 0.603 & $0.00 \%$ \\
\hline & Study design & & & & & \\
\hline & Hospital & 8 & 1.123 & $1.030-1.223$ & 0 & $74.60 \%$ \\
\hline & Population & 3 & 1.274 & $0.654-2.482$ & 0 & $91.10 \%$ \\
\hline & Genotyping method & & & & & \\
\hline & PCR-PFLP & 7 & 1.181 & $1.038-1.343$ & 0 & $84.80 \%$ \\
\hline & MassARRAY & 3 & 1.506 & $1.265-1.793$ & 0.364 & $1.00 \%$ \\
\hline \multirow[t]{11}{*}{ TrpTrp vs ArgArg } & Overall & 11 & 2.108 & $1.593-2.789$ & 0.095 & $38.20 \%$ \\
\hline & Overall in HWE & 5 & 2.783 & $2.114-3.664$ & 0.233 & $28.20 \%$ \\
\hline & Ethnicity & & & & & \\
\hline & Asian & 7 & 1.375 & $1.264-1.495$ & 0.114 & $41.50 \%$ \\
\hline & Caucasian & 3 & 1.191 & $0.655-2.167$ & 0.333 & $9.10 \%$ \\
\hline & Study design & & & & & \\
\hline & Hospital & 8 & 1.37 & $1.260-1.491$ & 0.147 & $35.30 \%$ \\
\hline & Population & 2 & 2.751 & $1.767-4.283$ & 0.309 & $3.40 \%$ \\
\hline & Genotyping method & & & & & \\
\hline & PCR-PFLP & 7 & 1.473 & $1.207-1.798$ & 0.005 & $67.40 \%$ \\
\hline & MassARRAY & 3 & 2.341 & $1.542-3.555$ & 0.749 & $0.00 \%$ \\
\hline \multirow[t]{11}{*}{ ArgTrp vs ArgArg } & Overall & 12 & 1.106 & $0.901-1.359$ & 0 & $76.00 \%$ \\
\hline & Overall in HWE & 5 & 1.779 & $1.032-3.066$ & 0 & $87.80 \%$ \\
\hline & Ethnicity & & & & & \\
\hline & Asian & 7 & 1.041 & $0.984-1.100$ & 0.527 & $0.00 \%$ \\
\hline & Caucasian & 4 & 0.903 & $0.806-1.013$ & 0.701 & $0.00 \%$ \\
\hline & Study design & & & & & \\
\hline & Hospital & 8 & 1.024 & $0.970-1.082$ & 0.242 & $23.50 \%$ \\
\hline & Population & 3 & 0.931 & $0.278-3.115$ & 0.002 & $89.50 \%$ \\
\hline & Genotyping method & & & & & \\
\hline & PCR-PFLP & 7 & 1.156 & $0.9587-1.396$ & 0 & $86.50 \%$ \\
\hline & MassARRAY & 3 & 1.28 & $1.025-1.598$ & 0.749 & $0.00 \%$ \\
\hline \multirow[t]{11}{*}{ TrpTrp vs ArgArg+ArgTrp } & Overall & 11 & 1.918 & $1.575-2.336$ & 0.42 & $2.30 \%$ \\
\hline & Overall in HWE & 5 & 2.288 & $1.758-2.979$ & 0.426 & $0.00 \%$ \\
\hline & Ethnicity & & & & & \\
\hline & Asian & 7 & 1.359 & $1.212-1.524$ & 0.092 & $45 \%$ \\
\hline & Caucasian & 3 & 1.205 & $0.662-2.191$ & 0.337 & $8.10 \%$ \\
\hline & Study design & & & & & \\
\hline & Hospital & 8 & 1.352 & $1.246-1.468$ & 0.122 & $38.70 \%$ \\
\hline & Population & 2 & 1.422 & $1.057-1.913$ & 0.384 & $0.00 \%$ \\
\hline & Genotyping method & & & & & \\
\hline & PCR-PFLP & 7 & 1.363 & $1.182-1.571$ & 0.082 & $46.50 \%$ \\
\hline & MassARRAY & 3 & 2.2 & $1.454-3.330$ & 0.768 & $0.00 \%$ \\
\hline \multirow[t]{11}{*}{ ArgTrp+TrpTrp vs ArgArg } & Overall & 12 & 1.23 & $0.997-1.519$ & 0 & $80.10 \%$ \\
\hline & Overall in HWE & 5 & 1.881 & $1.245-2.841$ & 0 & $84.10 \%$ \\
\hline & Ethnicity & & & & & \\
\hline & Asian & 7 & 1.098 & $1.043-1.156$ & 0.128 & $39.60 \%$ \\
\hline & Caucasian & 4 & 0.909 & $0.812-1.018$ & 0.647 & $0.00 \%$ \\
\hline & Study design & & & & & \\
\hline & Hospital & 8 & 1.091 & $1.006-1.183$ & 0.026 & $56 \%$ \\
\hline & Population & 3 & 1.469 & $0.476-4.538$ & 0 & $92.50 \%$ \\
\hline & Genotyping method & & & & & \\
\hline & PCR-PFLP & 7 & 1.16 & $0.999-1.346$ & 0 & $81.20 \%$ \\
\hline & MassARRAY & 3 & 1.44 & $1.173-1.768$ & 0.539 & $0.00 \%$ \\
\hline
\end{tabular}

*In general the fix-effects model was used, only when the $P_{h}<0.10$ random-effects model was used

\section{Discussion}

Glioma is generally considered to be a geneenviroment interaction disease, and a better understanding of the mechanism of glioma will help us find better ways to prevent, diagnose, or treat glioma. At present, notwithstanding some risk factors have been found, the etiology of glioma is still poorly understood (Kishida et al., 2012, Marumoto et al., 2012). However, as we all know that genetic factors play crucial roles in the occurrence of glioma (Melin, 2011; von Deimling et al., 2011). Confirmed of biomarkers of genetic factors could expect 
to make early diagnosis, predict patients outcome, or carry out individualized or personalized therapy. Unfortunately, up to now few genetic biomarkers has been identified as good biomarkers for glioma patients. To find some glioma relevant genetic biomarkers is of the most importance to improve the prognosis.

As we all know, DNA repair genes could maintain the genome integrity, and thus DNA repair genes polymorphisms are potential candidates which can modify the development of gliomas. XRCCl is one of the most important DNA repair genes responsible for BER pathway and DBS caused by IR. The XRCCI Arg194Trp polymorphism is located in an evolutionary conserved linker region, makes the chances of occurrence of chromosomal breaks highly increased (Monaco et al., 2007). Up to now, a number of articles have been performed to address the association between XRCC1 Arg194Trp polymorphism and the risk of gliomas, but yielded conflicting results. Because of the above-mentioned contradictory results from relatively small articles underpowered to detect the effects and previous meta-analysis indicated that XRCC1 Arg194Trp polymorphism was no association with the development of glioma an updated meta-analysis should be a proper way to obtain a more definitive conclusion.

Although previous meta-analyses have confirmed that the results did not showed any association between XRCC1 Arg194Trp polymorphism and glioma risk for all genetic models (Jiang et al., 2013), even in subgroup analyses based on the source of controls, ethnicity and histological subtype. After that, published data regarding the association between Arg 194Trp polymorphism and glioma risk were inconsistent. To derive a more accurate estimation of the association between XRCC1 Arg 194Trp polymorphism and glioma risk, we performed a meta-analysis. This meta-analysis, including a total of 5407 glioma cases and 7715 controls from 12 casecontrol studies, examined the potential association of the polymorphisms of the DNA repair gene XRCC1 Arg194Trp with glioma risk. We observed a fixed overall $25.9 \%$ increased risk of glioma for the Trp allel of the Arg194Trp polymorphism, compared with the wild allel (OR, 1.259; 95\%CI, 1.045-1.517). Similarly, for the Arg 194Trp, the variant genotypes (TrpTrp), compared with the wild-type homozygote (Arg/Arg), were associated with a significantly increased glioma risk (OR, 2.108; 95\%CI, 1.593-2.789) for ethnicity types without between-study heterogeneity. However, due to the presence of marginal statistical evidence and small sample size for Arg194Trp, our result as regards this polymorphism should always be regard as preliminary. However, our analysis shows that even if a general variant in the functional region of a conclusive meaningful gene had an effect on human disease, such as glioma, it may play only a tiny role in the development of glioma, which is conform to the characteristics of low-penetrance genes (Lohmueller et al., 2003).

Furthermore, three recent meta-analyses by Luo et al. (2013), Pan et al. (2013) and Xu et al. (2013) were evaluated the association between Arg194Trp polymorphism and glioma risk, which was basically in consist with our meta-analysis results that Arg194Trp polymorphism may contribute to the susceptibility of glioma, particularly in Asians, but not in Caucasians. It is notable that given the specific multiplicity of possible comparisons and the inescapable adaptation of choosing, associations may have been detected by chance alone. Some articles have been proposed for evaluating correlations between genetic polymorphisms and disease (Freely associating, 1999). The claim was that studies "ideally should have large sample sizes, small P values, report associations that make biological sense, and alleles that affect the gene product in a physiologically meaningful way" (Hu et al., 2005). The scientific hypotheses and sample size of the study are crucial to know the ratio of false-positive findings of meta-analysis that are attributable to constituent studies with selection bias from publication, poor study design, and nondifferential misclassification errors (Wacholder et al., 2002).

One study conducted in region of Europe with 700 glioma patients and 1556 controls reported that no association between the Arg194Trp polymorphism and glioma cancer risk (Kiuru et al., 2008). The other studies in USA consisted of a total sample size ( 1514 cases and 2755 controls) showed that Arg194Trp did not confer an effect on glioma (Liu et al., 2009; McKean-Cowdin et al., 2009; Rajaraman et al., 2010). Two of articles did not contain sex, age and other match statistic parameters, whereas since 2012, published articles included detailed statistic parameters such as smoking, drinking, cancer history of first relatives and IR exposure, which suggested that support the current meta-analysis that XRCC1 Arg194Trp may play a role in individual susceptibility to glioma.

In addition, between-study heterogeneity is a potential problem which was not avoidable. Despite several differences in the studies about ethnicity, sample sizes, source of controls, and genotyping method, we didn't observe significant heterogeneity between studies for the Arg 194Trp polymorphism. Importantly, we carefully performed sensitivity analysis according to sample size and leave-one-out analysis, conducted different conclusions with the previous meta-analysis. In view of this, the results of our meta-analysis, substantially, are sound and reliable.

Similar to other meta-analyses, our study also has a few potential limitations. First, owing to lack of adjusted variables the present meta-analysis was based primarily on unadjusted effect estimates and CIs, thus the effect estimates were relatively imprecise, a more accurate analysis could be conducted if adjusted variables were available in all articles. Second, quite small sample size existed for several subgroup analyses, such as source of controls from population. Third, glioma is known as a multifactor disease, due to lack of detailed data, such as environmental factors, physical inactivity and dietary state factors, thus the gene-gene and gene-environment interactions were not addressed in this meta-analysis. Fourth, several articles indicated that demographic parameters are not well adjusted statistically (Kiuru et al., 2008; McKean-Cowdin et al., 2009). Fifth, misclassifications of genotypes may also impact the results because cases were not verification by other gold standard 


\section{Cheng Xu et al}

methods in several studies, and the quality control of genotyping was also not well-verification in some articles. Lastly, although we did not discover publication bias, selection bias may exist because only literatures published in English were included.

In conclusion, our current study support that XRCC1 Arg194Trp polymorphism may contribute to individual susceptibility of glioma. To further evaluate genegene and gene- environment interactions on XRCC1 polymorphisms and glioma risk, thousands of subjects and tissue-specific biochemical characterizations are required.

\section{References}

Akbari M, Solvang-Garten K, Hanssen-Bauer A, et al (2010). Direct interaction between XRCC1 and UNG2 facilitates rapid repair of uracil in DNA by $X R C C 1$ complexes. $D N A$ Repair, 9, 785-95.

Bondy ML, Scheurer ME, Malmer B, et al (2008). Brain tumor epidemiology: consensus from the Brain Tumor Epidemiology Consortium. Cancer, 113, 1953-68.

Caldecott KW, Tucker JD, Stanker LH, Thompson LH (1995). Characterization of the XRCC1-DNA ligase III complex in vitro and its absence from mutant hamster cells. Nucleic Acids Res, 23, 4836-43.

Campalans A, Marsin S, Nakabeppu Y, et al (2005). XRCC1 interactions with multiple DNA glycosylases: a model for its recruitment to base excision repair. DNA Repair, 4, 826-35.

Connelly JM, Malkin MG (2007). Environmental risk factors for brain tumors. Curr Neurol Neurosci Rep, 7, 208-14.

Custodio AC,Almeida LO, Pinto GR, et al (2011). Analysis of the polymorphisms XRCC1 Arg194Trp and XRCC1Arg399Gln in gliomas. Genet Mol Res, 10, 1120-9.

Dianov GL, Prasad R, Wilson SH, Bohr VA (1999). Role of DNA polymerase beta in the excision step of long patch mammalian base excision repair. J Biol Chem, 274, 13741-3.

Freely associating (1999). Nat Genet, 22, 1-2.

Hu XB, Feng Z, Fan YC, Xiong ZY, Huang QW (2011). Polymorphisms in DNA repair gene $X R C C l$ and increased genetic susceptibility to glioma. Asian Pac J Cancer Prev, 12, 2981-4.

Hu Z, Ma H, Chen F, Wei Q, Shen H (2005). XRCC1 polymorphisms and cancer risk: a meta-analysis of 38 case-control studies. Cancer Epidemiol Biomarkers Prev, 14, 1810-8.

Jiang L, Fang X, Bao Y, et al (2013). Association between the $X R C C 1$ polymorphisms and glioma risk: a meta-analysis of case-control studies. PLoS One, 8, 55597.

Kishida Y, Natsume A, Toda H, et al (2012). Correlation between quantified promoter methylation and enzymatic activity of O6-methylguanine-DNA methyltransferase in glioblastomas. Tumour Biol, 33, 373-81.

Kiuru A, Lindholm C, Heinavaara S, et al (2008). XRCC1 and XRCC3 variants and risk of glioma and meningioma. $J$ Neurooncol, 88, 135-42.

Liu HB, Peng YP, Dou CW, et al (2012). Comprehensive study on associations between nine SNPs and glioma risk. Asian Pac J Cancer Prev, 13, 4905-8.

Liu Y, Scheurer ME, El-Zein R, et al (2009). Association and interactions between DNA repair gene polymorphisms and adult glioma. Cancer Epidemiol Biomarkers Prev, 18, 204-14.

Lohmueller KE, Pearce CL, Pike M, Lander ES, Hirschhorn JN (2003). Meta-analysis of genetic association studies supports a contribution of common variants to susceptibility to common disease. Nat Genet, 33, 177-82.
Luo KQ, Mu SQ, Wu ZX, Shi YN, Peng JC (2013). Polymorphisms in DNA repair genes and risk of glioma and meningioma. Asian Pac J Cancer Prev, 14, 449-52.

Marsin S, Vidal AE, Sossou M, et al (2003). Role of XRCC1 in the coordination and stimulation of oxidative DNA damage repair initiated by the DNA glycosylase hOGG1. J Biol Chem, 278, 44068-74.

Marumoto T, Saya H (2012). Molecular biology of glioma. Adv Exp Med Biol, 746, 2-11.

McKean-Cowdin R, Barnholtz-Sloan J, Inskip PD, et al (2009). Associations between polymorphisms in DNA repair genes and glioblastoma. Cancer Epidemiol Biomarkers Prev, 18, 1118-26.

Melin B (2011). Genetic causes of glioma: new leads in the labyrinth. Curr Opin Oncol, 23, 643-7.

Monaco R, Rosal R, Dolan MA, Pincus MR, Brandt-Rauf PW (2007). Conformational effects of a common codon 399 polymorphism on the BRCT1 domain of the XRCC1 protein. Protein J, 26, 541-6.

Ostrom QT, Barnholtz-Sloan JS (2011). Current state of our knowledge on brain tumor epidemiology. Curr Neurol Neurosci Rep, 11, 329-35.

Pan WR, Li G, Guan JH (2013). Polymorphisms in DNA repair genes and susceptibility to glioma in a chinese population. Int J Mol Sci, 14, 3314-24.

Rajaraman P, Hutchinson A, Wichner S, et al (2010). DNA repair gene polymorphisms and risk of adult meningioma, glioma, and acoustic neuroma. Neuro Oncol, 12, 37-48.

Ricard D, Idbaih A, Ducray F, et al (2012). Primary brain tumours in adults. Lancet, 379, 1984-96.

Schwartzbaum JA, Fisher JL, Aldape KD, Wrensch M (2006). Epidemiology and molecular pathology of glioma. Nat Clin Pract Neurol, 2, 494-503.

Thompson LH, West MG (2000). XRCC1 keeps DNA from getting stranded. Mutat Res, 459,1-18.

Vidal AE, Boiteux S, Hickson ID, Radicella JP (2001). XRCC1 coordinates the initial and late stages of DNA abasic site repair through protein-protein interactions. $E M B O J, \mathbf{2 0}$, 6530-9.

von Deimling A, Korshunov A, Hartmann C (2011). The next generation of glioma biomarkers: MGMT methylation, BRAF fusions and IDH1 mutations. Brain Pathol, 21, 74-87.

Wacholder S, Rothman N, Caporaso N (2002). Counterpoint: bias from population stratification is not a major threat to the validity of conclusions from epidemiological studies of common polymorphisms and cancer. Cancer Epidemiol Biomarkers Prev, 11, 513-20.

Wang D, Hu Y, Gong H, et al (2012). Genetic polymorphisms in the DNA repair gene $X R C C 1$ and susceptibility to glioma in a Han population in northeastern China: a case-control study. Gene, 509, 223-7.

Wen PY, Kesari S (2008). Malignant gliomas in adults. $N$ Engl J Med, 359, 492-507.

Xu G, Wang M, Xie W, Bai X (2013). Three polymorphisms of DNA repair gene $X R C C 1$ and the risk of glioma: a casecontrol study in northwest China. Tumour Biol.???

Zhou LQ, Ma Z, Shi XF, et al (2011). Polymorphisms of DNA repair gene $X R C C 1$ and risk of glioma: a case-control study in Southern China. Asian Pac J Cancer Prev, 12, 2547-50. 